\title{
Note sur l'application de la formule de Bernoulli aux courants liquides
}

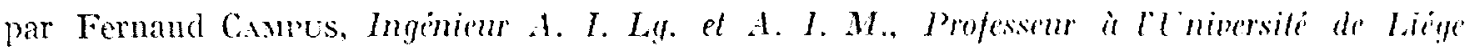

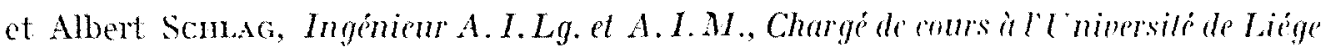

Là formule de Bernoulli se déduit de l'applicalion des équations générales d'Euler, au cas parliculier des liquides pesants. Il en résulte immédiatement que, t'n raison mème des hypothèses faites au cours de la démonstration de ces équations, la formule de Bernoulli ne peut s'appliquer qu'ì un mime filet liquide, c'està-dire aux points situés sur la trajectoire d'une mème particule, qu'il s'agisse de la trajectoire réelle, ainsi que cela résulle directement des équations dEuler ou de la trajectoire dans le mouvement moyen local, comme l'a démontré M. Boussinesq dans son Essai sur la théorie des eaux couranies.

On n'est donc pas en droit d'appliquer la formule de Bernoulli à l'ensemble d'une masse liquide en mouvement; le terme relatif à l'énergie cinétique doit nolamment, comme nous allons le voir, ètre affecté d'un facteur correctif. On sait, en effet, que la quantité de mouvement et l'énergie vive d'une masse liquide dont les particules possèdent des vitesses différentes, sont supérieures à celles qu'on déterminerail en utilisant la vitesse moyenne $\mathrm{U}$. On démontre que la quantilé de mouvement

$$
\because \int_{\omega} \cdot u^{2} \cdot d \omega=\left(1+r_{1}\right) ; \mathrm{U}^{2}(\omega)
$$

et que la force vive

$$
P \int_{01}^{\infty} u^{3} \cdot d(0)=x \cdot p \mathrm{C}^{3}(1)
$$

$r_{i}$ étant toujours positif et $\alpha$ elant toujours plus grand que l'unité et approximativement égal à $1+3 r$. En pratique, on prend souvent

$$
r_{1}=-0,037 \text { et } \%=1,11 \text { soit } 10 / 0
$$

11 règne dans la littérature lechnique, une grande confusion sur le point de savoir s'il faut introduire le facteur $\left(1+r_{i}\right)$ on le facteur $\alpha$ dans l'expression de la formule de Bernoulli élendue à une masse liquide tout entière.

Certains auteurs appliquent tout simplement la formule de Bernoulli sous sa forme initiale, à la masse entière, l'altitude $z$ étant celle du centre de gravité de la section normale aux filets (que nous appellerons dans ce qui suit, section transversale), la pression et la vitesse étant la pression et la vitesse moyennes, et le terme relatif à l'énergie cinctique n'ćtant affecté d'aucun facteur de correction.

D'autres, comme Eydoux (Hydraulique générale et appliquée) et Forchheimer (Hydraulik) ecrivent, - justement pour les cas de la pratique, mais sans insister sur une explication :

$$
\frac{\pi U^{2}}{2 g}+\frac{P}{z}+Z=- \text { Constante, }
$$

où Z est la cote du centre de gravilé de la section transversale, $\mathrm{U}$ et $p$ la vitesse et la pression moyennes.

Quant à Mouret, dans son Cours d'Hydraulique autographié (1913-14) de l'Ecole Nationale des Ponts et Chaussées, il déter- mine lenersie mécanique lobale du comans liquide par mile de poids

$$
\frac{\alpha \mathrm{U}^{2}}{2 y}+\frac{1}{\pi Q} \int \operatorname{pd} d \mathrm{Q}+z_{1}
$$

dans laquelle $z_{1}$ est l'ordonné du eentre de gravile. Il deduit de cetle expression la forme du theoreme de Bernoulli applicable aux courants liquides. en verlu do principe de la conservalion de linergie.

Enfin, Flamanl (Hydraulique, pp. 38 el suivantes de la $3^{\text {e }}$ édition) introduit le facteur $\left(1-r_{1}\right)$ au lien de $x$ dans son équalion générale du monroment permanent. Flamant suppose un courant. liquide défini par le parallélisme à peu près parfait des vilesses individuelles des divers filets; il peul ainsi mettre la premiere equation d'luler sous la forme :

$$
\frac{1}{2} \frac{d p}{d x}-g \cdot \sin \mathrm{I}-\mathrm{F}-u^{\prime}
$$

F, terme lenant comple des frotlements, disparail dans le cas d'un liquide parfail: daulle part, flamant demontre que $\frac{d p}{d x}$ est constant dans une meme section transversale et egat a $\frac{d p_{0}}{d x}$; il cn résulte que $u$ un $u \frac{d u}{d x}$ doil egalement dre constant dans la section. La vitesse variant sans discontinuité dans la section transversale, il existe un point ou la vilesse réelle est précisément égale à la vitesse moyenne $U$, dono :

$$
\frac{d\left(u^{2}\right)}{d x}=\frac{d\left(\mathrm{U}^{2}\right)}{d x}
$$

el. l'écuation devient :

$$
\frac{1}{p} \cdot \frac{d p_{0}}{d x}=-s \cdot \sin I-\frac{\left.d \mathrm{U}^{2}\right)}{d x}
$$

Or, l'équation finate qu'établit Flamant est, dans l'hypolhèse' d'un liquide parfait,

$$
\frac{1}{p} \cdot \frac{d p_{0}}{d x}=g \cdot \sin I-\left(1+r_{t}\right) \frac{d(\mathrm{U})}{d x}
$$

Comme $r_{i}$ est different de $O$, si la vitesse n'est pas identique en lous les points d'une section, il fant en conchure que

$$
\frac{d\left(U^{2}\right)}{d x}=0 \text {, on } \quad \mathrm{I} \frac{d \mathrm{C}}{d x}=0 \text { ou eneo e } \frac{d \mathrm{U}}{d x}=0
$$

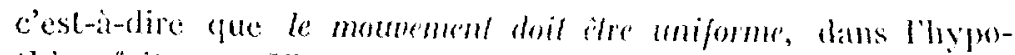
these faite par Flamant, d'un icoulement par lilets parallides.

La formule finale qu'on appliquerail a l'ecoulement d'un comrant en géneral, scrail donc entache d'une errent.

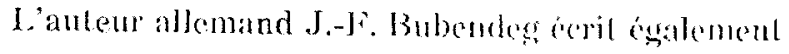

$$
\alpha=1+r_{i}
$$


De ce qui pricede, on peut conclure que la question vant la peine d'etre examince soigneusement.

Soit donc un liquide parfail en mouvement.

l'égualion de Bermoulli pouma elor appligué à chaque rilel, contre deux sections 1 el 2.

$$
z_{1}+\frac{p_{1}}{2}+\frac{u_{1}^{2}}{2 g}=z_{2}+\frac{p_{2}}{2}+\frac{u_{2}^{2}}{2 g}
$$

Mulliplions les deux mombres de retle egalite par le debit

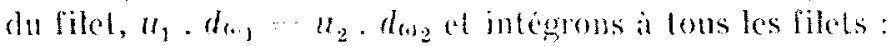

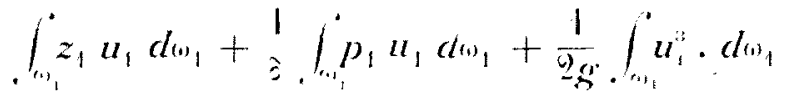

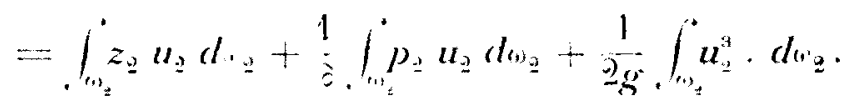

$\int_{d .} z u d(t)$ represente le moment par rapport au plan d'origine des z, de la surface (ne en chaque point de laquelle on supposerait rxister une densite massigue proportionnelle à $u$. \% étant le coulde de gravile de celle surface ainsi chargée,

$$
\int_{1, z}^{*} z \cdot u \cdot d n=Z[\omega
$$

$\int_{n}^{\infty} p a d w$ pent anssi se representer par $P L_{0,}, P$ etant en vertu da deuxieme principe de la movenne (1), une valeur intermediaire entre les pressions maximum et minimum dans la surface w. P' ast donc la pression réple regnant en un certain puint de la surfare.

linlin,

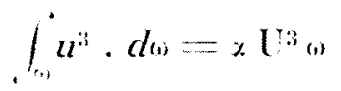

Ie theoreme de Bemoull devient ansi :

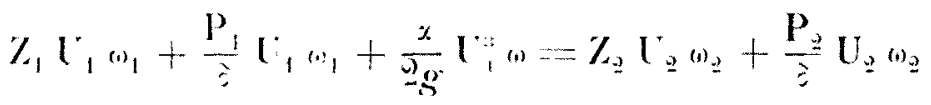

$$
\begin{aligned}
& +\frac{x}{2 g} l^{3}=
\end{aligned}
$$

ou en divisant par to debil tolal

$$
\begin{aligned}
& \mathrm{U}_{1, H_{1}}=\mathrm{U}_{\mathrm{g}} \\
& Z_{1}+\frac{P_{1}}{i}+x \frac{U_{1}}{g}=Z_{2}+\frac{P_{9}}{i}+x \frac{U_{2}}{2 g}=\text { Constante }
\end{aligned}
$$

(1) Si dans lintervalle $(a, b)$, la fonction $F(x)$ n'est jamais négative ni constamment mulle, et si la fonetion $\approx(x)$ a pour valeur maximum 11 et pour valeur minimum $m$, on $a$ :

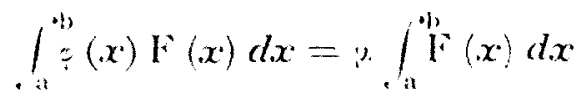

") désignant un ertain nombre compris entre $\mathrm{M}$ et $m$.

\section{Cas parteliters}

1. La pression varie hydrostaliquement dans la section transversale. -... Si nous désignons par $p_{0}$ la pression à l'altitude $z=0$,

$$
P=p_{0}-z \vdots
$$

el le deuxième terme de l'équalion devient:

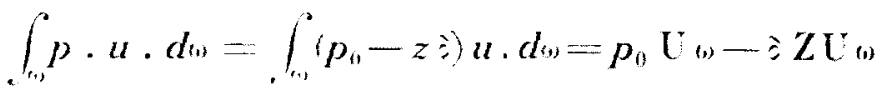

$$
\begin{aligned}
& =\left(p_{0}-2 Z\right) \mathrm{l}_{\omega}-\mathrm{P} \cdot \mathrm{P} \cdot \omega
\end{aligned}
$$

Pétant ici la pression au point riallitude Z, c'est-à-dire au centre de gravité de la surface (1) chargée comme nous l'avons indiqué plus haut.

$\mathrm{Si}$ du reste la pression varie hydrostaliquement, la somme $=+\frac{p}{z}$ est la mene en lous les points de la section.

Ce cas sera en particuliar celui qui se rencontre dans l'étude des courants, soil en conduites forcées, soit en canaux ouverts. L'équation de Bernoulli s'écrira alors :

$$
z+\frac{p}{3}+\frac{\mathrm{L}^{2}}{2 y}=\text { Conslante }
$$

z et p se rapportant d̀ un même point quelconque de la section transversale, soit son centre de gravite géométrique, soit un point de la surface libre, soil un point pris sur le fond du lit, etc.

II. Les vitesses et les pressions sont distribuées symétriquement par rapport à un axe horizontal silué dans la section transverversale. - On voit aisément que dans ce cas, $Z$ est l'altitude de cet axe horizontal, c'est-à-dire dans ce cas du centre de gravité géométrique de la section, tandis que $P$ est la pression en un certain point de la section, qui n'est pas mieux défini que dans le cas général.

C'est le cas des ronduiles verlicales, circulaires, rectangulaires, etc.

$$
* *
$$

En conclusion, laffirmation de Ph. Forchheimer que " dans Ies mouvements rotationnels, le théorème de Bernoulli s'applique aux différents filets, mais non pas à l'ensemble du courant " est ambiguë. On peut exprimer au contraire, que, abstraction faite des résistances des parois, si le théorème de Bernoulli s'applique aux différents filets, il s'applique aussi à l'ensemble du courant, par intégration se rapportant non aux sections élémentaires do des filets, mas à leurs débits élémentaires $u$. dw Il est done bien evident aussi que le terme $\frac{\mathrm{U}^{\mathrm{g}}}{\mathrm{gg}}$ doit ètre affecté du coefficient $x=1=3 r_{\text {, }}$ el non du facteur $\left(1+r_{1}\right)$. 